\title{
Recovery of the soliton self-frequency shift by optical phase conjugation
}

\author{
Sien Chi \\ Institute of Electro-Optical Engineering, National Chiao Tung University, 1001 Ta Hsueh Road, Hsinchu, Taiwan, China \\ Senfar Wen \\ Department of Electrical Engineering, Chung-Hua Polytechnic Institute, 30 Tung Shiang, Hsinchu, Taiwan, China
}

Received February 22, 1994

\begin{abstract}
The recovery of the self-frequency shift effect on a soliton by optical phase conjugation is investigated. It is proved that, without third-order dispersion and fiber loss, the soliton self-frequency shift can be completely recovered. The recoveries for a fundamental soliton and a second-order soliton in the presence of third-order dispersion and of fiber loss, which is compensated by the distributed erbium-doped fiber amplifiers, are numerically studied.
\end{abstract}

Recently optical phase conjugation (OPC) was used for the compensation for chromatic dispersion in a single-mode fiber. ${ }^{1,2}$ The compensation of chromatic dispersion by OPC was been proposed by Yariv et $a l$. in $1979 .{ }^{3}$ In fact, in addition to chromatic dispersion, the effect of delayed nonlinear response on an optical pulse can also be completely recovered by OPC in the lossless case. ${ }^{4}$ In Refs. 5 and 6 soliton interaction resulting from the nonlinear Kerr effect is shown to be undone by OPC with the fiber loss compensated by the optical amplifiers. Because the Ramaneffect-induced soliton self-frequency shift ${ }^{7}$ (SFS) is an effect of delayed nonlinear response, the shifts can also be recovered by OPC in the lossless case. In this Letter we numerically show recoveries of the effect of the SFS on the fundamental soliton and the second-order soliton when the effects of third-order dispersion and fiber loss are considered, where the fiber loss is compensated by distributed erbium-doped fiber amplifiers (DEDFA's) to reduce the soliton power perturbation.

The soliton propagating in the fiber follows the modified nonlinear Schrödinger equation ${ }^{8}$ (MNSE)

$$
\begin{aligned}
i \frac{\partial \phi}{\partial z}-\frac{1}{2} \beta_{2} \frac{\partial^{2} \phi}{\partial \tau^{2}}-i \frac{1}{6} \beta_{3} \frac{\partial^{3} \phi}{\partial \tau^{3}}+n_{2} \beta_{0}|\phi|^{2} \phi & \\
& -c_{r} \frac{\partial|\phi|^{2}}{\partial \tau} \phi=-\frac{1}{2} i \alpha \phi
\end{aligned}
$$

where the self-steepening term has been neglected for a pulse width $\gg 100 \mathrm{fs} ; \beta_{2}$ and $\beta_{3}$ represent the second- and third-order dispersion, respectively; $n_{2}$ is the Kerr coefficient; $c_{r}$ is the coefficient of the SFS; and $\alpha$ is the fiber loss. When $\beta_{3}=c_{r}=\alpha=0$, Eq. (1) becomes the nonlinear Schrödinger equation. For the nonlinear Schrödinger equation the pulse experiences only second-order dispersion and self-phase modulation, and there are bright $N$-soliton solutions for $\beta_{2}<0$, where $N$ is a positive integer. For the fundamental soliton $(N=1)$ the pulse shape does not change along the fiber. For the higher-order soliton $(N>1)$ the pulse shape varies periodically along the fiber. When the soliton pulse is short, the effects of third-order dispersion and the SFS become significant, and the MNSE should be used. The effect of third-order dispersion is also significant when the soliton wavelength is near the zero-dispersion wavelength. For the MNSE there are no soliton solutions. Third-order dispersion unsymmetrically distorts the soliton and decreases the group velocity of the soliton. ${ }^{9}$ The effect of the SFS downshifts the soliton frequency through the Raman effect and also asymmetrically distorts the soliton. ${ }^{7}$

Following the same procedures given by Ref. 4, one can easily prove the recovery of the effect of SFS by the OPC. Replacing the field $\phi$ by its complex conjugate $\phi^{*}$ and reversing the direction of propagation, $z \rightarrow-z$, yields Eq. (1) with $\beta_{3}=\alpha=0$ :

$$
\begin{aligned}
-i \frac{\partial \phi^{*}}{\partial z}-\frac{1}{2} \beta_{2} \frac{\partial^{2} \phi^{*}}{\partial \tau^{2}}+n_{2} \beta_{0}|\phi|^{2} \phi^{*} & \\
-c_{r} \frac{\partial|\phi|^{2}}{\partial \tau} \phi^{*} & =0 .
\end{aligned}
$$

Notice that Eq. (2) is exactly the complex conjugate of Eq. (1) with $\beta_{3}=\alpha=0$. Therefore these effects on the pulse, which propagates some distance, can be recovered by the conjugate pulse that propagates the same distance. However, because of third-order dispersion and fiber loss, the combined effect of secondorder dispersion, self-phase modulation, and the SFS cannot be recovered completely. In the following numerical examples are shown for the recovery of the combined effect in the fiber.

The soliton wavelength is assumed to be $1.55 \mu \mathrm{m}$, and the following numerical parameters are used: $\beta_{2}=-2.55 \mathrm{ps}^{2} / \mathrm{km}[2 \mathrm{ps} /(\mathrm{km} \mathrm{nm})], \beta_{3}=$ $0.074 \mathrm{ps}^{3} / \mathrm{km}, n_{2}=3.2 \times 10^{-20} \mathrm{~m}^{2} / \mathrm{W}$, and $c_{r}=$ $3.85 \times 10^{-16} \mathrm{ps} \mathrm{m} / \mathrm{W}$. The effective fiber cross section is $35 \mu \mathrm{m}^{2}$. The fiber loss is compensated by the DEDFA to reduce the effect of the power 
(a)
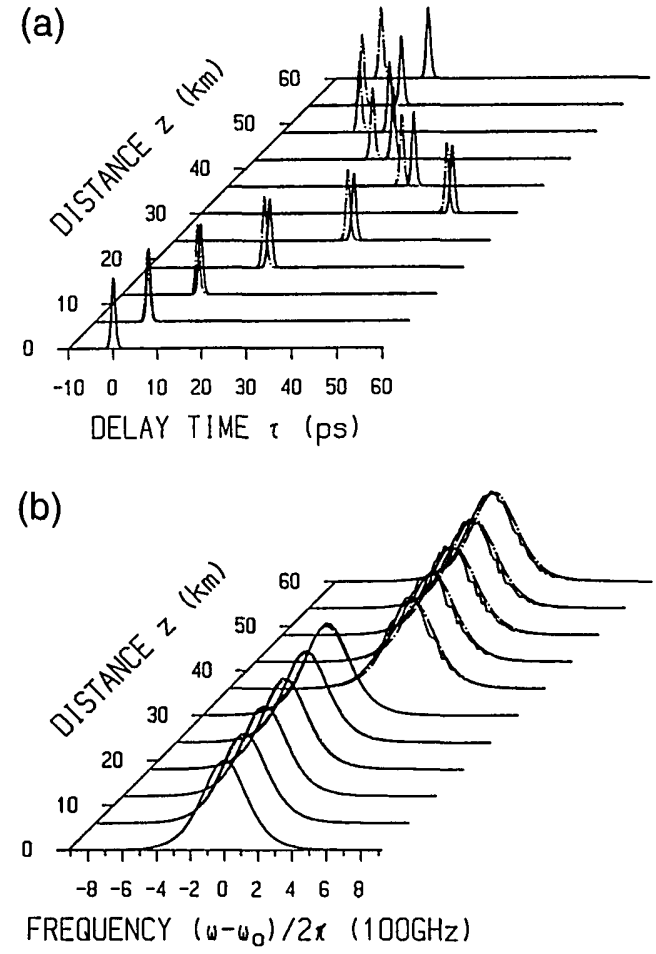

Fig. 1. (a) Power envelope and (b) power spectrum of the $N=1$ soliton with a pulse width $\tau_{\mathrm{W}}=1 \mathrm{ps}$ along two cascaded DEDFA's. The length of a DEDFA is $30 \mathrm{~km}$. An optical phase conjugator is placed between the two DEDFA's. The ideal case is shown by the dashed-dotted curves for comparison.

perturbation on the soliton transmission. ${ }^{10}$ The DEDFA is pumped bidirectionally, and its pump wavelength is $1.48 \mu \mathrm{m}$. Both the intrinsic fiber losses at the soliton wavelength and the pump wavelength are assumed to be $0.23 \mathrm{~dB} / \mathrm{km}$. The other parameters of the DEDFA are the same as those given in Ref. 10, except the doping density $N_{t}$ of the erbium-doped fiber, which is given in the following. To enhance the effect of the SFS, short soliton pulses are considered. The length of the amplifier is taken as $30 \mathrm{~km}$. Because the soliton period is only $0.2 \mathrm{~km}$ for the pulse width (FWHM) $\tau_{W}=1 \mathrm{ps,} \mathrm{a} \mathrm{small}$ power perturbation is required for stable soliton transmission. The maximum deviation $\Delta$ of the soliton power in the DEDFA and the required pump power $P_{p 0}$ depend on $N_{t}$ and the average power of the solitons. With $\tau_{W}=1 \mathrm{ps}$ and a $100-\mathrm{MHz}$ repetition rate, the minimum $\Delta=1.65 \%$ for $N_{t}=$ $2.6 \times 10^{21} \mathrm{~m}^{-3}$, and the corresponding $P_{p 0}=$ $26.8 \mathrm{~mW}$. For larger $N_{t}, P_{p 0}$ decreases and $\Delta$ increases. For the range of the soliton power considered in this Letter, the corresponding $\Delta$ 's are approximately $1.6 \%, 10 \%$, and $20 \%$ for $N_{t}=2.6 \times 10^{21}$, $8.4 \times 10^{21}$, and $1.14 \times 10^{22} \mathrm{~m}^{-3}$, respectively.

Figures 1(a) and 1(b) show the power envelope and the power spectrum of the $N=1$ soliton with $\tau_{W}=1 \mathrm{ps}$ along two cascaded DEDFA's, respectively, for $N_{t}=2.6 \times 10^{21} \mathrm{~m}^{-3}$. An optical phase conjugator is placed between the DEDFA's, i.e., at $30 \mathrm{~km}$ in the figure. The conjugator is assumed to be ideal, and the operation of the conjugator is to take the complex conjugate of the field of the soliton from the first DEDFA. In the figure the ideal case with $\beta_{3}=\alpha=0$ is shown by the dashed-dotted curves for comparison. It is shown that in the first DEDFA the soliton downshifts its frequency, and its group velocity decreases. After the conjugator, the spectrum of the soliton is inverted with respect to the original carrier frequency $\omega_{0}$. For the ideal case, after propagating through the second DEDFA in the frequency domain the soliton downshifts its frequency to $\omega_{0}$, and its spectrum is restored; in the time domain the soliton moves back without changes in the delay time and pulse shape. Thus, the restoration of the soliton is complete for the ideal case. For the case in the presence of third-order dispersion and power perturbation in the frequency domain, the soliton almost downshifts its frequency to $\omega_{0}$, but its spectrum distorts; in the time domain the pulse distorts only slightly but has a net change of the delay time $\delta \tau$. The net change in the delay time is due to both third-order dispersion and power perturbation. The power perturbation contributes to the net change in the delay time because the soliton SFS changes with the soliton power. Figure 2 shows $\delta \tau$ versus $\tau_{W}$ for various $N_{t}$ 's, where the cases without third-order dispersion $\left(\beta_{3}=0\right)$ are also shown for comparison. One can see that $\delta \tau$ increases as $\Delta$ increases but decreases as $\tau_{W}$ increases. The net change in the delay time is due mainly to third-order dispersion. When $\Delta$ is small the restorations of the pulse shapes are better.

For the higher-order soliton the change in the pulse shape along the fiber is more serious and the restoration of the soliton by OPC is more difficult. To reduce the effect of third-order dispersion, we take a longer pulse in the example. Figures 3(a) and 3(b) show the power envelope and the power spectrum of the $N=2$ soliton with $\tau_{W}=5$ ps along two cascaded DEDFA's, respectively, for $N_{t}=2.6 \times 10^{21} \mathrm{~m}^{-3}$. The optical phase conjugator is also placed between the DEDFA's. In Fig. 3, the ideal case with $\beta_{3}=\alpha=0$ is shown by the dashed-dotted curves for comparison. One can see that within the first DEDFA the soliton splits into a small blue-shifted pulse and a

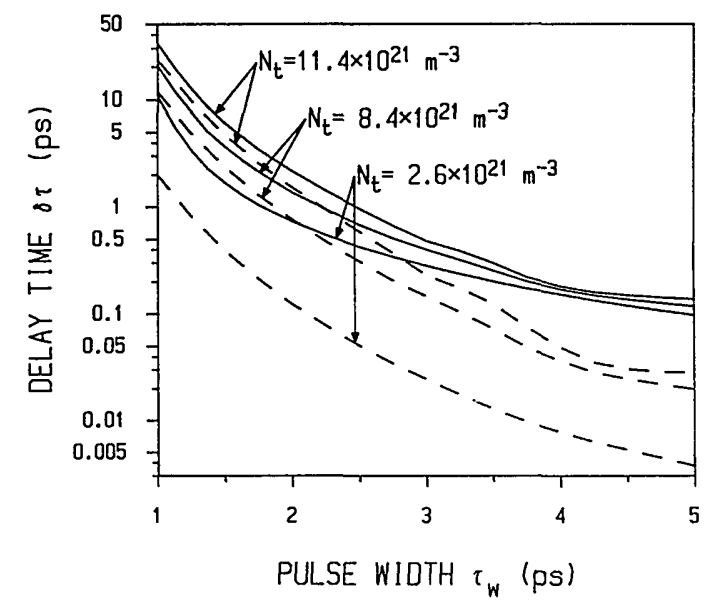

Fig. 2. Net change of the delay time $\delta \tau$ of the $N=1$ soliton versus pulse width $\tau_{W}$ for various doping densities $N_{t}$ 's. The cases with $\beta_{3}=0$ are shown by the dashed curves. 
(a)

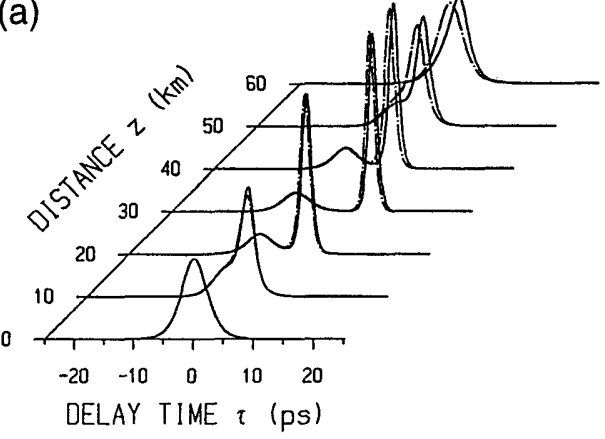

(b)

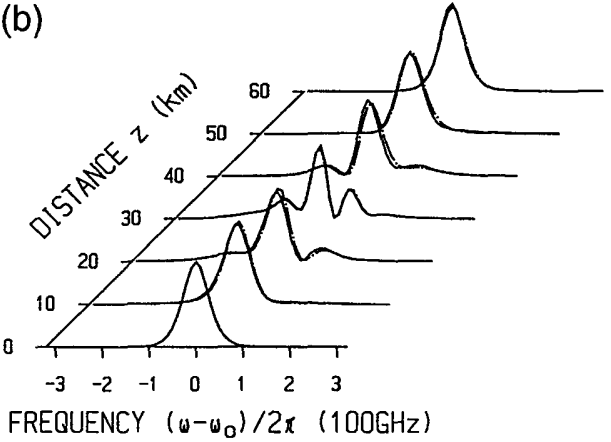

Fig. 3. (a) Power envelope and (b) power spectrum of the $N=2$ soliton with a pulse width $\tau_{W}=5$ ps along two cascaded DEDFA's. The length of a DEDFA is $30 \mathrm{~km}$. An optical phase conjugator is placed between the two DEDFA's. The ideal case is shown by the dashed-dotted curves for comparison.

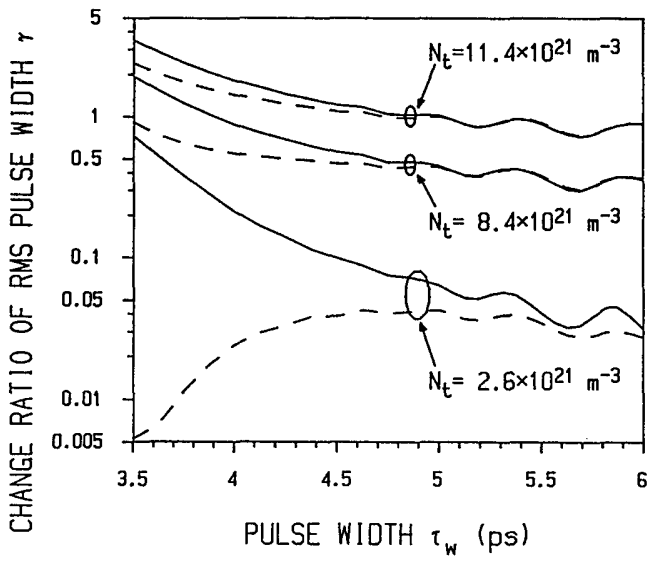

Fig. 4. Change ratio $\gamma$ of the rms pulse width of the $N=2$ soliton at the output of the second DEDFA versus the pulse width $\tau_{W}$ for various doping densities $N_{t}$ 's. The cases with $\beta_{3}=0$ are shown by the dashed curves.

large red-shifted pulse. The two generated pulses move apart. After the conjugator, the two pulses come back and recombine. For the ideal case the restoration of the $N=2$ soliton is complete. In the presence of third-order dispersion and power perturbation the restoration is not complete but is still good. In the frequency domain the soliton spectrum also recovers well. If the soliton propagates a longer distance before the conjugator, the restoration is not so good because the two pulses cannot come back in time to recombine. Figure 4 shows the change ratio $\gamma$ of the rms pulse width of the $N=2$ soliton at the output of the second DEDFA versus $\tau_{W}$ for various
$N_{t}$ 's, where the cases with $\beta_{3}=0$ are also shown for comparison. When $\beta_{3}=0$ the recoveries are better. When $\beta_{3} \neq 0$ and $\tau_{W}$ is less than approximately $3.5 \mathrm{ps}$ the two pulses cannot come back in time to recombine. One can see that $\gamma$ increases as $\Delta$ increases but decreases as $\tau_{W}$ increases. For the cases with $N_{t}=8.4 \times 10^{21}$ and $1.14 \times 10^{22} \mathrm{~m}^{-3}$ shown in Fig. 4, the restorations are not good even when $\tau_{W}=6 \mathrm{ps}$ because the power perturbations are high.

Notice that the OPC used above is assumed to be ideal. The conjugation can be achieved by four-wave mixing (FWM) with the pump frequency at $\omega_{0}$. For the cases shown in Figs. 1 and 3 the FWM should be backward ${ }^{3}$; otherwise the spectrum of the conjugate soliton cannot be separated from the spectra of the input soliton and the pump beam. Recent experiments show that a large frequency inversion can be obtained by use of forward FWM. ${ }^{1,2}$ In Ref. 1 forward FWM is achieved in the dispersion-shifted fiber where the pump wavelength is at the zerodispersion wavelength. In Ref. 2 forward FWM is achieved in the semiconductor laser amplifier. Generation of a phase-conjugate soliton is more feasible with a semiconductor laser amplifier when the pulse width is short because the required fiber length of the dispersion-shifted fiber is long, and the soliton may be further distorted. If the spectrum of the conjugate soliton can be separated from the spectra of the input soliton and the pump beam with an optical filter, forward FWM with the pump frequency at $\omega_{0}$ can be used. On the other hand, because the soliton frequency is inverted by the conjugator, it is advantageous to use the conjugator to maintain the soliton wavelength in the gain band of the amplifier, which is used to compensate for the fiber loss.

In conclusion, the recovery of the effect of the SFS on a soliton by OPC is considered. It is proved that, without third-order dispersion and fiber loss, such an effect can be completely recovered. In the presence of third-order dispersion and fiber loss, which is compensated by the DEDFA's, the restorations of the solitons are shown and discussed.

This research is supported by National Science Council of the Republic of China under contract NSC 83-0417-E009-013.

\section{References}

1. S. Watanabe, T. Naito, and T. Chikama, IEEE Photon. Technol. Lett. 5, 92 (1993).

2. M. C. Tatham, G. Sherlock, and L. D. Westbrook, Electron. Lett. 29, 1851 (1993).

3. A. Yariv, D. Fekete, and D. M. Pepper, Opt. Lett. 4, 52 (1979).

4. R. A. Fisher, B. R. Suydam, and D. Yevick, Opt. Lett. 8, 611 (1983).

5. W. Forysiak and N. J. Doran, Electron. Lett. 30, 154 (1994).

6. S. Wen and S. Chi, Electron. Lett. 30, 663 (1994).

7. J. P. Gordon, Opt. Lett. 11, 662 (1986).

8. G. P. Agrawal, Nonlinear Fiber Optics (Academic, Boston, Mass., 1989), Chap. 5.

9. S. Wen and S. Chi, Opt. Quantum Electron. 21, 335 (1989).

10. S. Wen, Opt. Lett. 19, 22 (1994). 